\title{
Anti-glutamate Dehydrogenase Antibody Positive Cerebellar Ataxia and Stiff Person Syndrome Responding to Dual Treatment with Steroids and Intravenous Immunoglobulin: A Case Presentation and Literature Review
}

\author{
Furkan M. Yilmaz $^{1}$, Dena Little ${ }^{1}$, Micheal Gallagher ${ }^{2}$, Amy Colcher ${ }^{1}$ \\ 1. Neurology, Cooper Neurological Institute, Cooper University Hospital, Camden, USA 2. \\ Neurophysiology, University of Maryland Medical Center, Baltimore, USA
}

$\square$ Corresponding author: Furkan M. Yilmaz, mdfurkanyilmaz@yahoo.com

Disclosures can be found in Additional Information at the end of the article

\section{Abstract}

Anti-glutamic acid decarboxylase (anti-GAD) antibody syndrome (aGAS) has various presentations including cerebellar ataxia (CA) and stiff person syndrome (SPS). This is a treatable cause of CA and SPS. We present a case of a 49-year-old man who developed blurred vision, slurred speech, difficulty walking, unsteady gait, and clumsiness which had progressed over four months. The patient was found to have anti-GAD ab (+) CA and SPS and experienced significant symptomatic improvements after treatment with intravenous (IV) steroids followed by intravenous immunoglobulin (IVIG). The patient's improvement persisted when he was reevaluated at follow up one month later. Since anti-GAD ab related diseases, including antiGAD CA and SPS, are rarely diagnosed, there is limited data regarding the treatment of this condition. As there are only a few cases in the literature similar to this one, highlighting the successful treatment of anti-GAD ab cerebellar ataxia and SPS with dual therapy (steroids followed by IVIG) is important.

Received 05/12/2019

Review began 05/27/2019

Review ended 05/30/2019

Published 06/06/2019

(C) Copyright 2019

Yilmaz et al. This is an open access article distributed under the terms of the Creative Commons Attribution License CC-BY 3.0., which permits unrestricted use, distribution, and reproduction in any medium, provided the original author and source are credited.
Categories: Endocrinology/Diabetes/Metabolism, Neurology, Rheumatology

Keywords: hypothyroidism, cerebellar ataxia, steroids, intravenous immunoglobulins, scale for the assessment and rating of ataxia (sara), methylprednisolone, type 2 diabetes mellitus, glutamic acid decarboxylase antibody, stiff-person syndrome

\section{Introduction}

Glutamic acid decarboxylase (GAD) is an enzyme which catalyzes the conversion of glutamate to gamma-aminobutyric acid (GABA). GAD is expressed in the central nervous system (CNS), pancreas, and thyroid gland [1]. GAD is an intracellular enzyme [2]. The mechanism of cell destruction with an extracellular antibody, and the role of the antibody in the pathophysiology of the disease, are not clear [3]. Animal studies give promising evidence for a possible mechanism of the disease [4]. Anti-GAD antibody (Ab) cerebellar ataxia is a rare cause of subacute, gait-predominant ataxia [5]. Most of the affected patients are women, many of whom also have Type 1 diabetes mellitus (DM) [3]. The diagnosis is made by the presence of elevated anti-GAD antibody levels in the serum or cerebrospinal fluid (CSF) [6]. Currently, there is limited data, primarily in the form of case reports, regarding the treatment of this condition.

\section{How to cite this article}

Yilmaz F M, Little D, Gallagher M, et al. (June 06, 2019) Anti-glutamate Dehydrogenase Antibody Positive Cerebellar Ataxia and Stiff Person Syndrome Responding to Dual Treatment with Steroids and Intravenous Immunoglobulin: A Case Presentation and Literature Review. Cureus 11(6): e4851. DOI 


\section{Case Presentation}

A 49-year-old plumber presented to the hospital with blurred vision, slurred speech, difficulty walking, unsteady gait, and clumsiness which had progressed over four months. He suffered a fall while at work. He had back pain and noticed imbalance and difficulty with his gait.

On examination, he was alert and oriented with mild pseudo-bulbar effect. Extra-ocular movements displayed saccadic overshoot. His speech was dysarthric and slow. Cranial nerves were intact. There was increased tone in the thoracolumbar paraspinal muscles. Deep tendon reflexes were $1+$, and plantar responses were flexor bilaterally. There was dysdiadochokinesia, and all extremities demonstrated dysmetria. The patient had postural instability with a positive Romberg sign. His gait was wide-based and ataxic, and he was unable to ambulate more than a few steps due to imbalance. His score on the scale for the assessment and rating of ataxia (SARA) [7] was 12.

Basic labs as well as vitamin b12, angiotensin-converting enzyme level, antineutrophil cytoplasmic antibodies, myeloperoxidase antibodies, proteinase $3 \mathrm{~b}$ antibodies, vitamin $\mathrm{E}$ level, ceruloplasmin, HIV 1 and 2, celiac disease panel, inflammatory markers (erythrocyte sedimentation rate (ESR) and c-reactive protein (CRP)), rheumatologic antibodies, and heavy metal screen were negative. Cerebrospinal fluid (CSF) analysis (cell count, protein, and glucose) were within normal limits. CSF oligoclonal band and CSF serology were negative.

Paraneoplastic antibody testing including anti-Hu, anti-Yo, anti-Ri, anti-Ma was negative. CSF anti-GAD was positive with a titer of $>250$ international units per milliliter (IU/Ml). Magnetic resonance imaging (MRI) of the brain did not show an acute or chronic disease process. Computed tomography scan (CT) of the chest, abdomen, and pelvis was negative for any underlying malignancy. Prostate-specific antigen (PSA) was negative.

Intravenous methylprednisolone (500 mg twice a day) was initiated. The patient's back pain significantly improved within 24 hours of the five-day course. By the end of the steroid course, his appendicular ataxia improved; however, his gait remained ataxic. Therefore, we initiated additional treatment with IVIG ( $2 \mathrm{mg}$ per kg divided into a five-day course). By day five of the IVIG course, the patient's ataxic gait significantly improved, and he was able to ambulate beyond 100 feet. By the day of discharge, his SARA score had improved to eight. Pre- and posttreatment videos of his neurological examination were taken. One month after the treatment, the patient was able to walk at least 100 feet without the help of a cane or walker.

\section{Discussion}

GAD catalyzes the conversion of glutamate to GABA, and antibodies to this enzyme can lead to a multitude of neurologic syndromes including, but not limited to, limbic encephalitis, stiff person syndrome, refractory epilepsy, and cerebellar ataxia [8-9]. Anti-GAD antibody syndrome (aGAS) can present with thyroiditis, pernicious anemia, vitiligo, and type one diabetes [10-12]. In addition to hypothyroidism, our patient was diagnosed with type 2 diabetes mellitus, shortly before the diagnosis of aGAS. This may raise a question regarding the role of anti-GAD antibodies in insulin resistance as well [13]. A thorough neurological exam should be done including cranial nerve, cerebellar, and gait exam in a patient with ataxia and stiff person symptoms. Saccadic overshoot can be due to the cerebellar effects of the disease process [14]. Various ophthalmic manifestations of aGAS including vertical nystagmus [15], opsoclonus [1416], and defects in smooth pursuit [16] can be seen.

The results of the anti-GAD test are essential as quantitative data (in our case anti-GAD>250 $\mathrm{IU} / \mathrm{Ml}$ ). Even low anti-GAD levels can present with clinical features like upbeat nystagmus (<30 $\mathrm{IU} / \mathrm{Ml})$ and type $1 \mathrm{DM}(<100 \mathrm{IU} / \mathrm{Ml})$ [12]. The anti-GAD titers are expected to be high for stiff person syndrome (SPS) or for cerebellar ataxia (CA) [17]. After diagnoses of most autoimmune 
CAs, a paraneoplastic origin must be ruled out, especially for the patients with subacute symptom onset. But for the cases with anti-GAD associated CA, this may not be necessary as concurrent malignancy is rare [18]. Antibodies to gliadin and transglutaminase need to be checked as there is an overlap between gluten ataxia and aGAS ataxia. A strict gluten diet may improve the ataxia in this subgroup regardless of detectable enteropathy [10]. Brain imaging can be helpful to differentiate subacute vs. chronic disease as the latter one can present with cerebellar atrophy on an MRI brain scan.

Given the postulation that this is strictly an autoimmune-mediated disease, treatment tends to consist of immune modulating therapies [19]. Despite given developing literature, there is no standard of care when it comes to the treatment of anti-GAD cerebellar ataxia. Treatment typically consists of glucocorticoids, IVIG, and plasmapheresis [20]. There is limited data regarding the time course of such treatments and if combining the treatments is of any benefit. Corticosteroids have been tried as the first-line therapy for anti-GAD associated neurologic disorders [20]. Additional case reports suggest that a combination of IVIG and delayed plasmapheresis show efficacy regarding symptomatic improvement [12, 19]. For maintenance therapy, it has been recommended that oral steroids, IVIG, azathioprine, and mycophenolate mofetil may be reasonable options [20]. Data also suggests that these treatments are more beneficial in acute/subacute presentations of anti-GAD cerebellar ataxia [11]. If the treatment is not providing symptomatic relief, an alternative treatment method should be tried. For our patient, we chose initially to treat with five days of high-dose IV steroids followed by a five-day course of IVIG. This regimen had resulted in a significant improvement in the patient's symptoms and SARA score by day five of the IVIG course. There are other cases in the literature that show that the steroid and IVIG combination was used with variable outcomes [19].

The clinical course, prognosis, and the response to therapy are different in various patients. Age less than 60 years, subacute presentation, the absence of atrophy on brain imaging, and low anti-GAD titers were predictive of good outcomes [4].

The limitations of our study include that we did not check anti-GAD index, which is a ratio test between blood and CSF [17]. This test would have confirmed the intrathecal vs. systemic production of antibodies. We also did not repeat the anti-GAD antibody test during follow up visits, which would have helped to see the response of the antibody to the treatment [13].

\section{Conclusions}

We presented a case with anti-GAD ab positive cerebellar ataxia, which we treated with a combination of a five-day IV steroid course immediately followed by IVIG treatment. This treatment regimen resulted in a significant improvement in the patient's symptoms and functionality. More studies are needed to determine if the treatment regimen we chose will be as effective in other patients with the same condition.

\section{Appendices}




\section{Cureus}

\begin{tabular}{|c|c|c|}
\hline Test & Value & Reference Range and Units \\
\hline Vitamin b12 & 727 & $211-911 \mathrm{pg} / \mathrm{mL}$ \\
\hline Rapid plasma reagin (RPR) & Nonreactive & Nonreactive \\
\hline Angiotensin-converting enzyme level & 20 & $9-67 \mathrm{U} / \mathrm{L}$ \\
\hline Glutamic acid decarboxylase & $>250$ & $<5[I U] / m L$ \\
\hline Lactate & 0.7 & $0.5-2.2 \mathrm{mmol} / \mathrm{L}$ \\
\hline Pyruvate & 0.88 & $0.30-1.50 \mathrm{mg} / \mathrm{dL}$ \\
\hline Vitamin E level & 17.6 & $5.7-19.9 \mathrm{mg} / \mathrm{L}$ \\
\hline Ceruloplasmin & 20 & $18-36 \mathrm{mg} / \mathrm{dL}$ \\
\hline Copper & 86 & $70-175 \mathrm{ug} / \mathrm{dL}$ \\
\hline Human Immune Deficiency Virus (HIV) 1 and 2 & Nonreactive & Nonreactive \\
\hline Erythrocyte sedimentation rate (ESR) & 6 & $0-15 \mathrm{~mm} / \mathrm{h}$ \\
\hline C-reactive protein (CRP) & $<0.03$ & $<0.50 \mathrm{mg} / \mathrm{dL}$ \\
\hline Hemoglobin A1c & 5.9 & $<5.7 \%$ \\
\hline Thyroid stimulating hormone (TSH) & 1.26 & $0.27-4.20 \mathrm{u}[\mathrm{IU}] / \mathrm{mL}$ \\
\hline Prostate specific antigen (PSA) & 0.14 & $0.0-4.0 \mathrm{ng} / \mathrm{mL}$ \\
\hline
\end{tabular}

\section{TABLE 1: Blood Workup}

pg: picograms; ml: milliliters; U: unit; IU: international units; mmol: millimoles; mg: milligrams; L: liter; dL: deciliters; ug: micrograms; mm: millimeters; ng: nanograms 


\section{Cureus}

\section{Test}

Rheumatologic antibodies

Thyroid peroxidase (TPO) Ab

Immunoglobulin A

Antineutrophil cytoplasmic antibodies

Myeloperoxidase antibodies

Proteinase $3 b$ antibodies

Anti-Sjögren's-syndrome-related antigen A (SSA) (RO) antibody

Anti-Sjögren's-syndrome-related antigen B (SSB) (LA) antibody

Tissue transglutaminase antibody b, immunoglobulin A

Lyme immunoglobulin $\mathrm{G} / \mathrm{Immunoglobulin} \mathrm{M}$ antibody

Neuronal nuclear antibody (Ri) Hu and Yo, CSF

Anti Ma and TA

Anti glutamic acid decarboxylase antibody
Value

Negative

403

200

Negative

Negative

Negative

$<1.0$ negative

$<1.0$ negative

1

$<0.9$

Negative

Negative

250.0
Reference Range and Units

Negative

$<9 \mathrm{IU} / \mathrm{mL}$

$80-463 \mathrm{mg} / \mathrm{dL}$

Negative

Negative

Negative

$<1.0$ negative [index_val]

$<1.0$ negative [index_val]

$<4$ [index_val]

0.19 [index_val]

Negative

Negative

$0.0-5 \mathrm{IU} / \mathrm{mL}$

\section{TABLE 2: Antibody Screening}

Please note high anti-glutamic acid decarboxylase antibody titers. IU: International unit, $\mathrm{mL}$ : milliliters, mg: milligram, $\mathrm{dL}$ : deciliters, index val: index value

\section{Imaging Modality Impression}

MRI brain $\quad$ Unremarkable contrast-enhanced MRI scan of the brain

CT chest abdomen pelvis

No primary malignancy or metastatic disease identified in the chest, abdomen, and pelvis

US testis

1. Calcifications in the scrotum likely representing scrotal pearls, the largest one in the right scrotal measuring approximately $0.4 \mathrm{~cm}$

2. The remainder of the examination is unremarkable

\section{TABLE 3: Imaging Studies}

MRI: magnetic resonance imaging, CT: computed tomography, US: ultrasonography 


\section{Cureus}

\begin{tabular}{lll} 
Test & Value & Reference Range and Units \\
\hline CSF appearance & & CLEAR AND COLORLESS \\
CSF nucleated cells & $0-5 / \mathrm{uL}$ & 0 \\
CSF red blood cells & $<1 / \mathrm{uL}$ & 13 \\
CSF corrected nucleated cells & $0-5 / \mathrm{uL}$ & $<1$ \\
CSF glucose & $40-75 \mathrm{mg} / \mathrm{dL}$ & 68 \\
CSF lactate dehydrogenase & $\mathrm{U} / \mathrm{L}$ & 23 \\
CSF protein & $15-45 \mathrm{mg} / \mathrm{dL}$ & 47 \\
CSF The Venereal Disease Research Laboratory test (VDRL) & Nonreactive & Nonreactive \\
CSF angiotensin-converting enzyme & $<=15 \mathrm{U} / \mathrm{L}$ & 8 \\
CSF cytology & Negative for malignant cells
\end{tabular}

\section{TABLE 4: CSF Analysis}

CSF: cerebrospinal fluid; pg: picograms; ml: milliliters; U: unit; IU: international units; mmol: millimoles; mg: milligrams; L: liter; dL: deciliters; ug: micrograms; mm: millimeters; ng: nanograms

\begin{tabular}{|l|l|l|}
\hline \hline Test & Value & Reference Range and Units \\
\hline Ferritin & 78 & $15-400 \mathrm{ng} / \mathrm{mL}$ \\
\hline Iron & 67 & $42-135 \mathrm{ug} / \mathrm{dL}$ \\
\hline Iron binding capacity & 271 & $225-425 \mathrm{ug} / \mathrm{dL}$ \\
\hline \% transferrin saturation & 25 & $15-50 \%$ \\
\hline TAB: deciliters; ug: micrograms; mm: millimeters; ng: nanograms & \\
\hline \hline
\end{tabular}




\section{Cureus}

\begin{tabular}{|c|c|c|}
\hline Test & Value & Reference Range and Units \\
\hline Blood arsenic & $<3$ & $<23$ ug/L \\
\hline Blood lead & $<1$ & $<5$ ug/dL \\
\hline Blood mercury & $<4$ & $<=10 \mathrm{ug} / \mathrm{L}$ \\
\hline Blood manganese & 1.1 & $<1.2 \mathrm{ug} / \mathrm{L}$ \\
\hline
\end{tabular}

\section{TABLE 6: Heavy Metal Screening}

$\mathrm{dL}$ : deciliters; ug: micrograms; mm: millimeters

\section{Additional Information Disclosures}

Human subjects: Consent was obtained by all participants in this study. Conflicts of interest: In compliance with the ICMJE uniform disclosure form, all authors declare the following: Payment/services info: All authors have declared that no financial support was received from any organization for the submitted work. Financial relationships: All authors have declared that they have no financial relationships at present or within the previous three years with any organizations that might have an interest in the submitted work. Other relationships: All authors have declared that there are no other relationships or activities that could appear to have influenced the submitted work.

\section{References}

1. Iwasaki H, Sato R, Shichiri M, Hirata Y: A patient with type 1 diabetes mellitus and cerebellar ataxia associated with high titer of circulating anti-glutamic acid decarboxylase antibodies. Endocr J. 2001, 48:261-268. 10.1507/endocrj.48.261

2. Honnorat J, Saiz A, Giometto B, et al.: Cerebellar ataxia with anti-glutamic acid decarboxylase antibodies: study of 14 patients. Arch Neurol. 2001, 58:225-230. 10.1001/archneur.58.2.225

3. Manto M, Mitoma H, Hampe CS: Anti-GAD antibodies and the cerebellum: where do we stand?. Cerebellum. 2018, 18:153-156. 10.1007/s12311-018-0986-6

4. Manto MU, Hampe CS, Rogemond V, Honnorat J: Respective implications of glutamate decarboxylase antibodies in stiff person syndrome and cerebellar ataxia. Orphanet J Rare Dz. 2011, 6:3. 10.1186/1750-1172-6-3

5. Dalakas MC: The role of IVIg in the treatment of patients with stiff person syndrome and other neurological diseases associated with anti-GAD antibodies. J Neurol. 2005, 252:19-25. 10.1007/s00415-005-1105-4

6. Vianello M, Tavolato B, Armani M, Giometto B: Cerebellar ataxia associated with antiglutamic acid decarboxylase autoantibodies. Cerebellum. 2003, 2:77-79. 10.1080/14734220309432

7. Schmitz-Hübsch T, du Montcel ST, Baliko L, et al.: Scale for the assessment and rating of ataxia: development of a new clinical scale. Neurol. 2006, 66:1717-1720. 10.1212/01.wnl.0000219042.60538.92

8. Mitoma H, Manto M, Hampe CS: Immune-mediated cerebellar ataxias: practical guidelines and therapeutic challenges. Curr Neuropharmacol. 2018, 17:33-58. 10.2174/1570159X16666180917105033

9. Pittock SJ, Yoshikawa H, Ahlskog JE, et al.: Glutamic acid decarboxylase autoimmunity with brainstem, extrapyramidal, and spinal cord dysfunction. Mayo Clin Proc. 2006, 81:1207-1214. 


\subsection{5/81.9.1207}

10. Proudfoot M, Wilkins A: Treatment of cerebellar ataxia in the context of systemic diseases . Curr Treat Options Neurol. 2017, 19:47. 10.1007/s11940-017-0485-y

11. Manto M, Hampe CS: Endocrine disorders and the cerebellum: from neurodevelopmental injury to late-onset ataxia. Handb Clin Neurol. 2018, 155:353-368. 10.1016/B978-0-44464189-2.00023-8

12. Nakajima H, Nakamura $Y$, Inaba $Y$, et al.: Neurologic disorders associated with anti-glutamic acid decarboxylase antibodies: a comparison of anti-GAD antibody titers and time-dependent changes between neurologic disease and type I diabetes mellitus. J Neuroimmunol. 2018, 317:84-89. 10.1016/j.jneuroim.2018.01.007

13. Folli F, Daniele G, Werner S, et al.: Membranous nephropathy and cerebellar degeneration with anti-GAD antibodies in type 2 diabetes mellitus. Acta Diabetol. 2015, 52:897-903. 10.1007/s00592-015-0728-x

14. Shaikh AG, Wilmot G: Opsoclonus in a patient with increased titers of anti-GAD antibody provides proof for the conductance-based model of saccadic oscillations. J Neurol Sci. 2016, 362:169-173. 10.1016/j.jns.2016.01.038

15. Baizabal-Carvallo JF, Alonso-Juarez M: Vertical nystagmus associated with glutamic acid decarboxylase antibodies responding to cyclophosphamide. J Neuroimmunol. 2018, 317:5-7. 10.1016/j.jneuroim.2018.01.013

16. Ghia T, Kanhangad M, Alessandri AJ, Price G, Gera P, Nagarajan L: Opsoclonus-myoclonus syndrome, neuroblastoma, and insulin-dependent diabetes mellitus in a child: a unique patient. Pediatr Neurol. 2016, 55:68-70. 10.1016/j.pediatrneurol.2015.09.021

17. Saiz A, Blanco Y, Sabater L, et al.: Spectrum of neurological syndromes associated with glutamic acid decarboxylase antibodies: diagnostic clues for this association. Brain. 2008, 131:2553-2563. 10.1093/brain/awn183

18. Bataller L, Valero C, Díaz R, et al.: Cerebellar ataxia associated with neuroendocrine thymic carcinoma and GAD antibodies. BMJ J Neurol Neurosurg Psychiatry. 2009, 80:696-697.

10.1136/jnnp.2008.161042

19. Georgieva Z, Parton M: Cerebellar ataxia and epilepsy with anti-GAD antibodies: treatment with IVIG and plasmapheresis. BMJ Case Rep. 2014, 2014:2013202314. 10.1136/bcr-2013202314

20. Ariño H, Gresa-Arribas N, Blanco Y, et al.: Cerebellar ataxia and glutamic acid decarboxylase antibodies: immunologic profile and long-term effect of immunotherapy. JAMA Neurol. 2014, 71:1009-1016. 10.1001/jamaneurol.2014.1011 